Modern Physics Letters A, Vol. 16, No. 9 (2001) 557-569

(c) World Scientific Publishing Company

\title{
BLACK HOLE PHYSICS, CONFINING SOLUTIONS OF SU(3)-YANG-MILLS EQUATIONS AND RELATIVISTIC MODELS OF MESONS
}

\author{
YU. P. GONCHAROV \\ Theoretical Group, Experimental Physics Department, State Technical University \\ Sankt-Petersburg 195251, Russia
}

Received December 2000

\begin{abstract}
The black hole physics techniques and results are applied to find the set of the exact solutions of the SU(3)-Yang-Mills equations in Minkowski spacetime in the Lorentz gauge. All the solutions contain only the Coulomb-like or linear in $r$ components of $\mathrm{SU}(3)$-connection. This allows one to obtain some possible exact and approximate solutions of the corresponding Dirac equation that can describe the relativistic bound states. Possible application to the relativistic models of mesons is also outlined.
\end{abstract}

\section{Introductory Remarks}

The present paper is motivated by the part of hadronic physics studying mesons. As is known, mesons within the hadron physics are the one of central sources of information about the quark interaction. Since nearly all known mesons are bound states of a quark $q$ and an antiquark $\overline{q^{\prime}}$ (where the flavours of $q$ and $\overline{q^{\prime}}$ may be different) then actually all modern meson spectroscopy is based on one or another quark model of mesons. Referring for more details, e. g., to the recent up-to-date review $t$ and references therein, it should be noted here that at present some generally accepted relativistic model of mesons is absent. The description of mesons in quark models is actually implemented by nonrelativistic manner (for example, on the basis of the Schrödinger equation) and then one tries to include relativistic corrections in one or another way. Such an inclusion is not single-valued and varies in dependence of the point of view for different authors (see, e. g. Refs. 3 and references therein). It would be more consistent, to our mind, building a primordially relativistic model so that one can then pass on to the nonrelativistic one by the standard limiting transition and, as a result, to estimate the relativistic effects in self-consistent way.

As follows from the main principles of quantum chromodynamics (QCD), the appropriate relativistic models for description of mesons as relativistic bound states of quarks should consist in considering the solutions of Dirac equation in a $\mathrm{SU}(3)$ Yang-Mills field representing gluonic field. The latter should be the solution of the corresponding Yang-Mills equations and should model the quark confinement. Such solutions are usually supposed to contain at least the components of the mentioned $\mathrm{SU}(3)$-field which are Coulomb-like or linear in $r$, the distance between quarks, and 
in what follows we call these solutions the confining ones. No general recipe of obtaining such solutions exists at present. In the given paper for this aim we shall employ the techniques used in Refs 3 for finding the $\mathrm{U}(\mathrm{N})$-monopole solutions in black hole physics and, to analyse the corresponding Dirac equation, we shall use the results of Refs 1 about spinor fields on black holes.

Further we shall deal with the metric of the flat Minkowski spacetime $M$ that we write down (using the ordinary set of local spherical coordinates $r, \vartheta, \varphi$ for spatial part) in the form

$$
d s^{2}=g_{\mu \nu} d x^{\mu} \otimes d x^{\nu} \equiv d t^{2}-d r^{2}-r^{2}\left(d \vartheta^{2}+\sin ^{2} \vartheta d \varphi^{2}\right) .
$$

As is known, such a metric can be obtained from the Schwarzschild black hole metric when the black hole mass is equal to 0 . Besides we have $|\delta|=\left|\operatorname{det}\left(g_{\mu \nu}\right)\right|=\left(r^{2} \sin \vartheta\right)^{2}$ and $0 \leq r<\infty, 0 \leq \vartheta<\pi, 0 \leq \varphi<2 \pi$.

Throughout the paper we employ the system of units with $\hbar=c=G=1$. Finally, we shall denote $L_{2}(F)$ the set of the modulo square integrable complex functions on any manifold $F$ furnished with an integration measure while $L_{2}^{n}(F)$ will be the $n$-fold direct product of $L_{2}(F)$ endowed with the obvious scalar product.

\section{Dirac equation}

To formulate the Dirac equation needed to us, let us notice that the relativistic wave function of meson can be chosen in the form

$$
\psi=\left(\begin{array}{c}
\psi_{1} \\
\psi_{2} \\
\psi_{3}
\end{array}\right)
$$

with the four-dimensional spinors $\psi_{j}$ representing $j$-th colour component of meson. Under this situation, if denoting $S(M)$ and $\xi$, respectively, the standard spinor bundle and three-dimensional vector one (equipped with a $\mathrm{SU}(3)$-connection) over Minkowski spacetime, we can construct tensorial product $S(M) \otimes \xi$. It is clear that $\psi$ is just a section of the latter bundle. Then the corresponding Dirac equation for $\psi$ can be obtained with using the construction of Dirac operator $\mathcal{D}$ with coefficients in $\xi$ and may look as follows

$$
\mathcal{D} \psi=\mu_{0} \psi
$$

where $\mu_{0}$ is the reduced relativistic mass which is equal, e. g., for quarkonia, to one half a constituent mass of quarks forming quarkonium, but definition of $\mu_{0}$ is, generally speaking, not single-valued and requires the specification within the concrete problem (see Ref. 13 ). Under this sitiation the coordinate $r$ makes sense of the distance between quarks.

From general considerations相 6 the explicit form of the operator $\mathcal{D}$ in local coordinates $x^{\mu}$ on a $2 k$-dimensional (pseudo)riemannian manifold can be written as follows

$$
\mathcal{D}=i\left(\gamma^{e} \otimes I_{3}\right) E_{e}^{\mu}\left(\partial_{\mu} \otimes I_{3}-\frac{1}{2} \omega_{\mu a b} \gamma^{a} \gamma^{b} \otimes I_{3}-i g A_{\mu}\right), a<b
$$


where $A=A_{\mu} d x^{\mu}, A_{\mu}=A_{\mu}^{c} T_{c}$ is a $\mathrm{SU}(3)$-connection in the bundle $\xi, I_{3}$ is the unit matrix $3 \times 3$, the matrices $T_{c}$ form a basis of the Lie algebra of $\mathrm{SU}(3)$ in 3-dimensional space (we consider $T_{a}$ hermitean which is acceptable in physics), $c=1, \ldots, 8, \otimes$ here means tensorial product of matrices, $g$ is a gauge coupling constant. Further, the forms $\omega_{a b}=\omega_{\mu a b} d x^{\mu}$ obey the Cartan structure equations $d e^{a}=\omega_{b}^{a} \wedge e^{b}$ with exterior derivative $d$, while the orthonormal basis $e^{a}=e_{\mu}^{a} d x^{\mu}$ in cotangent bundle and dual basis $E_{a}=E_{a}^{\mu} \partial_{\mu}$ in tangent bundle are connected by the relations $e^{a}\left(E_{b}\right)=\delta_{b}^{a}$. At last, matrices $\gamma^{a}$ represent the Clifford algebra of the corresponding quadratic form in $\mathbb{C}^{2^{k}}$. Below we shall deal only with $4 \mathrm{D}$ lorentzian case (quadratic form $Q_{1,3}=x_{0}^{2}-x_{1}^{2}-x_{2}^{2}-x_{3}^{2}$ ). For this we take the following choice for $\gamma^{a}$

$$
\gamma^{0}=\left(\begin{array}{cc}
1 & 0 \\
0 & -1
\end{array}\right), \gamma^{b}=\left(\begin{array}{cc}
0 & \sigma_{b} \\
-\sigma_{b} & 0
\end{array}\right), b=1,2,3,
$$

where $\sigma_{b}$ denote the ordinary Pauli matrices. It should be noted that, in lorentzian case, Greek indices $\mu, \nu, \ldots$ are raised and lowered with $g_{\mu \nu}$ of (1) or its inverse $g^{\mu \nu}$ and Latin indices $a, b, \ldots$ are raised and lowered by $\eta_{a b}=\eta^{a b}=\operatorname{diag}(1,-1,-1,-1)$, so that $e_{\mu}^{a} e_{\nu}^{b} g^{\mu \nu}=\eta^{a b}, E_{a}^{\mu} E_{b}^{\nu} g_{\mu \nu}=\eta_{a b}$ and so on.

Using the fact that all bundles over Minkowski spacetime are trivial and, as a result, they can be trivialized over the chart of local coordinates $(t, r, \vartheta, \varphi)$ covering almost the whole Minkowski manifold, we can concretize the Dirac equation (2) on the given chart for $\psi$ in the case of metric (1). Namely, we can put $e^{0}=d t$, $e^{1}=d r, e^{2}=r d \vartheta, e^{3}=r \sin \vartheta d \varphi$ and, accordingly, $E_{0}=\partial_{t}, E_{1}=\partial_{r}, E_{2}=\partial_{\vartheta} / r$, $E_{3}=\partial_{\varphi} /(r \sin \vartheta)$. This entails

$$
\omega_{12}=-d \vartheta, \omega_{13}=-\sin \vartheta d \varphi, \omega_{23}=-\cos \vartheta d \varphi .
$$

As for the connection $A_{\mu}$ in bundle $\xi$ then the suitable one should be the confining solution of the Yang-Mills equations

$$
\begin{gathered}
d F=F \wedge A-A \wedge F, \\
d * F=* F \wedge A-A \wedge * F+J
\end{gathered}
$$

with the exterior differential $d=\partial_{t} d t+\partial_{r} d r+\partial_{\vartheta} d \vartheta+\partial_{\varphi} d \varphi$ in coordinates $t, r, \vartheta, \varphi$ while the curvature matrix (field strentgh) for $\xi$-bundle is $F=d A+A \wedge A$ and $*$ means the Hodge star operator conforming to metric (1), $J$ is a source. It is clear that (6) is identically satisfied - this is just the Bianchi identity holding true for any connection (see, e. g., Refs. 6 ) so that it is necessary to solve only the equations (7). Introducing the Hodge star operator $*$ on 2-forms $F=F_{\mu \nu}^{a} T_{a} d x^{\mu} \wedge d x^{\nu}$ with the values in the Lie algebra of $\mathrm{SU}(3)$ by the relation (see, e. g., Ref.D)

$$
\left(F_{\mu \nu}^{a} d x^{\mu} \wedge d x^{\nu}\right) \wedge\left(* F_{\alpha \beta}^{a} d x^{\alpha} \wedge d x^{\beta}\right)=\left(g^{\mu \alpha} g^{\nu \beta}-g^{\mu \beta} g^{\nu \alpha}\right) F_{\mu \nu}^{a} F_{\alpha \beta}^{a} \sqrt{\delta} d x^{0} \wedge \cdots \wedge d x^{3}
$$

written in local coordinates $x^{\mu}$ [there is no summation over $a$ in (8)], in coordinates $t, r, \vartheta, \varphi$ we have the relations

$$
*(d t \wedge d r)=-r^{2} \sin \vartheta d \vartheta \wedge d \varphi, *(d t \wedge d \vartheta)=\sin \vartheta d r \wedge d \varphi,
$$




$$
\begin{gathered}
*(d t \wedge d \varphi)=-\frac{1}{\sin \vartheta} d r \wedge d \vartheta, *(d r \wedge d \vartheta)=\sin \vartheta d t \wedge d \varphi, \\
*(d r \wedge d \varphi)=-\frac{1}{\sin \vartheta} d t \wedge d \vartheta, *(d \vartheta \wedge d \varphi)=\frac{1}{r^{2} \sin \vartheta} d t \wedge d r,
\end{gathered}
$$

so that $*^{2}=* *=-1$, as should be for the manifolds with lorentzian signature.

Besides the sought solutions are usually believed to obey an additional gauge condition. In the present paper we take the Lorentz gauge condition that can be written in the form $\operatorname{div}(A)=0$, where the divergence of the Lie algebra valued 1-form $A=A_{\mu}^{c} T_{c} d x^{\mu}$ is defined by the relation (see, e. g. Refs.

$$
\operatorname{div}(A)=\frac{1}{\sqrt{|\delta|}} \partial_{\mu}\left(\sqrt{|\delta|} g^{\mu \nu} A_{\nu}\right)
$$

\section{Confining solutions}

Now we can use the techniques of Refs 3 to find a set of the confining solutions of Eq. (7). The essence of those techiniques consists in systematic usage of the Hodge star operator. Let firstly $J=0$ and let us put $T_{c}=\lambda_{\text {fo }}$ where $\lambda_{c}$ are the Gell-Mann matrices (whose explicit form can be found in Refs.3), and in detail let us write out the addend $A_{\mu}=A_{\mu}^{c} T_{c}$ of the operator $\mathcal{D}$ of (3)

$$
A_{\mu}^{c} \lambda_{c}=\left(\begin{array}{ccc}
A_{\mu}^{3}+\frac{1}{\sqrt{3}} A_{\mu}^{8} & A_{\mu}^{1}-i A_{\mu}^{2} & A_{\mu}^{4}-i A_{\mu}^{5} \\
A_{\mu}^{1}+i A_{\mu}^{2} & -A_{\mu}^{3}+\frac{1}{\sqrt{3}} A_{\mu}^{8} & A_{\mu}^{6}-i A_{\mu}^{7} \\
A_{\mu}^{4}+i A_{\mu}^{5} & A_{\mu}^{6}+i A_{\mu}^{7} & -\frac{2}{\sqrt{3}} A_{\mu}^{8}
\end{array}\right) .
$$

Then it is naturally to put $A_{\mu}^{c}=0$ with $c=1,2,4,5,6,7$ and to obtain from (2) the following system of Dirac equations for colour components $\psi_{j}$

$$
\begin{gathered}
i \gamma^{e} E_{e}^{\mu}\left[\partial_{\mu}-\frac{1}{2} \omega_{\mu a b} \gamma^{a} \gamma^{b}-i g\left(A_{\mu}^{3}+\frac{1}{\sqrt{3}} A_{\mu}^{8}\right)\right] \psi_{1}=\mu_{0} \psi_{1} \\
i \gamma^{e} E_{e}^{\mu}\left[\partial_{\mu}-\frac{1}{2} \omega_{\mu a b} \gamma^{a} \gamma^{b}-i g\left(-A_{\mu}^{3}+\frac{1}{\sqrt{3}} A_{\mu}^{8}\right)\right] \psi_{2}=\mu_{0} \psi_{2} \\
i \gamma^{e} E_{e}^{\mu}\left[\partial_{\mu}-\frac{1}{2} \omega_{\mu a b} \gamma^{a} \gamma^{b}-i g\left(-\frac{2}{\sqrt{3}} A_{\mu}^{8}\right)\right] \psi_{3}=\mu_{0} \psi_{3}
\end{gathered}
$$

Further for to avoid unnecessary complications with the Lorentz condition, it is simpler of all to put $A_{r, \vartheta}^{3,8}=0$. After this we search for the solution of (7) in the form $A=A_{t}(r) d t+A_{\varphi}(r) d \varphi$ with $A_{t, \varphi}=A_{t, \varphi}^{3} \lambda_{3}+A_{t, \varphi}^{8} \lambda_{8}$. It is then easy to check that $A \wedge A=0, F=d A=-\partial_{r} A_{t} d t \wedge d r+\partial_{r} A_{\varphi} d r \wedge d \varphi$ and, with the help of the relations (9), *F= $r^{2} \sin \vartheta \partial_{r} A_{t} d \vartheta \wedge d \varphi-\frac{1}{\sin \vartheta} \partial_{r} A_{\varphi} d t \wedge d \vartheta$. From here it follows that $* F \wedge A=A \wedge * F$ and Eq. (7) will be equivalent to the equation $d * F=0$ while the latter yields

$$
\partial_{r}\left(r^{2} \partial_{r} A_{t}\right)=0, \partial_{r}^{2} A_{\varphi}=0
$$


and we write down the solutions of (15) in the combinations that are just needed to insert into (12)-(14)

$$
\begin{gathered}
A_{t}^{3}+\frac{1}{\sqrt{3}} A_{t}^{8}=-\frac{a_{1}}{r}+A_{1}, \\
-A_{t}^{3}+\frac{1}{\sqrt{3}} A_{t}^{8}=\frac{a_{1}+a_{2}}{r}-\left(A_{1}+A_{2}\right), \\
-\frac{2}{\sqrt{3}} A_{t}^{8}=-\frac{a_{2}}{r}+A_{2}, \\
A_{\varphi}^{3}+\frac{1}{\sqrt{3}} A_{\varphi}^{8}=b_{1} r+B_{1}, \\
-A_{\varphi}^{3}+\frac{1}{\sqrt{3}} A_{\varphi}^{8}=-\left(b_{1}+b_{2}\right) r-\left(B_{1}+B_{2}\right), \\
-\frac{2}{\sqrt{3}} A_{\varphi}^{8}=b_{2} r+B_{2}
\end{gathered}
$$

with some constants $a_{j}, A_{j}, b_{j}, B_{j}$ parametrizing solutions.

Another class of the confining solutions of (7) can be obtained with the aid of the ansatz $A=A_{t}(r) d t+A_{\varphi}(r, \vartheta) d \varphi$, i. e. now we consider the component $A_{\varphi}$ depending also on $\vartheta$. Again we shall have $A \wedge A=0, F=d A=-\partial_{r} A_{t} d t \wedge d r+$ $\partial_{r} A_{\varphi} d r \wedge d \varphi+\partial_{\vartheta} A_{\varphi} d \vartheta \wedge d \varphi$ and $* F=r^{2} \sin \vartheta \partial_{r} A_{t} d \vartheta \wedge d \varphi-\frac{1}{\sin \vartheta} \partial_{r} A_{\varphi} d t \wedge d \vartheta+$ $\frac{1}{r^{2} \sin \vartheta} \partial_{\vartheta} A_{\varphi} d t \wedge d r$. Then anew $* F \wedge A=A \wedge * F$ and Eq. (7) is converted into $d * F=0$ that entails

$$
\begin{gathered}
\partial_{r}\left(r^{2} \partial_{r} A_{t}\right)=0 \\
r^{2} \partial_{r}^{2} A_{\varphi}+\sin \vartheta \partial_{\vartheta}\left(\frac{1}{\sin \vartheta} \partial_{\vartheta} A_{\varphi}\right)=0 .
\end{gathered}
$$

It is clear that Eq. (18) gives the same solutions of (16). We shall not here discuss the general form of the solution for Eq. (19) and only write out the possible solution of it which is useful to us in the present paper in the form slightly modifying (17)

$$
\begin{gathered}
A_{\varphi}^{3}+\frac{1}{\sqrt{3}} A_{\varphi}^{8}=-K_{1} \cos \vartheta+b_{1} r+B_{1}, \\
-A_{\varphi}^{3}+\frac{1}{\sqrt{3}} A_{\varphi}^{8}=\left(K_{1}+K_{2}\right) \cos \vartheta-\left(b_{1}+b_{2}\right) r-\left(B_{1}+B_{2}\right), \\
-\frac{2}{\sqrt{3}} A_{\varphi}^{8}=-K_{2} \cos \vartheta+b_{2} r+B_{2}
\end{gathered}
$$

with some real constants $K_{j}, b_{j}, B_{j}$ parametrizing solution.

Finally, we shall adduce one solution of (7) with a source. It is given by relations of (16) for $A_{t}$ while for $A_{\varphi}$ we have

$$
A_{\varphi}=I_{3}[(\mathbf{b} r+\mathbf{B}) \sin \vartheta+\mathbf{K} \cos \vartheta]
$$

with vectors $\mathbf{b}=\left(b_{1},-b_{1}-b_{2}, b_{2}\right), \mathbf{B}=\left(B_{1},-B_{1}-B_{2}, B_{2}\right), \mathbf{K}=\left(-K_{1}, K_{1}+\right.$ $\left.K_{2},-K_{2}\right)$. The conforming source is $J=-\frac{1}{r^{2} \sin ^{2} \vartheta} I_{3}(\mathbf{b} r+\mathbf{B}) d t \wedge d r \wedge d \vartheta$ and since 
$* J=\mathfrak{j}=\mathfrak{j}_{\mu} d x^{\mu}=\mathfrak{j}_{\varphi} d \varphi$ with $\mathfrak{j}_{\varphi}=-\frac{1}{r^{2} \sin \vartheta} I_{3}(\mathbf{b} r+\mathbf{B}), \operatorname{div}(\mathfrak{j})=0$, we can interpret $\mathfrak{j}$ as the colour current giving rise to the corresponding linear interaction.

At last, it is easy to check that all the solutions obtained satisfy the Lorentz gauge condition.

\section{Spectrum of bound states in the Coulomb-like case}

Returning to the Eqs. (12)-(14), we should note that when inserting the confining solutions gained into them the variables $r$ and $\vartheta$ cannot be, generally speaking, separated. Let us take, for example, the solutions of (16) and (21) for the YangMills equations (7) with the source $J$ described late in Sec. 3 (where we at first put $K_{j}=0$ ) and for all three equations let us employ the ansatz

$$
\psi_{j}=e^{i \omega_{j} t} r^{-1}\left(\begin{array}{c}
F_{j 1}(r) \Phi_{j}(\vartheta, \varphi) \\
F_{j 2}(r) \sigma_{1} \Phi_{j}(\vartheta, \varphi)
\end{array}\right), j=1,2,3
$$

with a $2 \mathrm{D}$ spinor $\Phi_{j}=\left(\begin{array}{c}\Phi_{j 1} \\ \Phi_{j 2}\end{array}\right)$. Then, in the way analogous to that of Refs.l, we can get, e. g., from (12) the system

$$
\begin{array}{r}
{\left[\left(\partial_{r}+\frac{1}{r}\right)+\frac{1}{r} \mathcal{D}_{0}-\sigma_{2} g\left(b_{1}+\frac{B_{1}}{r}\right)\right] \frac{1}{r} F_{11} \Phi_{1}=i\left(\mu_{0}-c_{1}\right) \frac{1}{r} F_{12} \Phi_{1},} \\
{\left[\left(\partial_{r}+\frac{1}{r}\right)+\frac{1}{r} \mathcal{D}_{0}-\sigma_{2} g\left(b_{1}+\frac{B_{1}}{r}\right)\right] \frac{1}{r} F_{12} \sigma_{1} \Phi_{1}=-i\left(\mu_{0}+c_{1}\right) \frac{1}{r} F_{11} \sigma_{1} \Phi_{1}}
\end{array}
$$

with $c_{1}=\omega_{1}-g\left(-a_{1} / r+A_{1}\right)$ while

$$
\mathcal{D}_{0}=\sigma_{1} \sigma_{2} \partial_{\vartheta}+\frac{1}{\sin \vartheta} \sigma_{1} \sigma_{3}\left(\partial_{\varphi}-\frac{1}{2} \sigma_{2} \sigma_{3} \cos \vartheta\right)
$$

is the euclidean Dirac operator on the unit sphere $\mathbb{S}^{2}$. It is not complicated to check that at $b_{1} \neq 0, B_{1} \neq 0$ the variables $r$ and $\vartheta$ are not separated. Under this situation we shall at first restrict ourselves to the case $b_{j}=B_{j}=0$ since under the circumstances we can use the results of Refs. 0 for to solve Eqs. (12)-(14) exactly. Primarily let us put also $K_{j}=0$ and then we shall modify results to take into account the case $K_{j} \neq 0$. For all three equations we employ the ansatz (22) and in the way analogous to that of Refs. 1 , we can obtain from (12)-(14) the systems

$$
\begin{aligned}
& \left(\partial_{r}+\frac{\lambda_{j}}{r}\right) F_{j 1}=i\left(\mu_{0}-c_{j}\right) F_{j 2}, \\
& \left(\partial_{r}-\frac{\lambda_{j}}{r}\right) F_{j 2}=-i\left(\mu_{0}+c_{j}\right) F_{j 1}
\end{aligned}
$$

with an eigenvalue $\lambda_{j}$ for the eigenspinor $\Phi_{j}$ of the above operator $\mathcal{D}_{0}, \lambda_{j}= \pm\left(l_{j}+\right.$ $1) \in \mathbb{Z} \backslash\{0\}, l_{j}=0,1,2 \ldots$ (for more details see Refs. 6 ). Besides

$$
c_{2}=\omega_{2}-g\left[\frac{a_{1}+a_{2}}{r}-\left(A_{1}+A_{2}\right)\right]
$$




$$
c_{3}=\omega_{3}-g\left(-\frac{a_{2}}{r}+A_{2}\right),
$$

so that the energy spectrum $\varepsilon$ of meson is given by the relation $\varepsilon=\omega_{1}+\omega_{2}+\omega_{3}$. The explicit form of $\Phi_{j}$ is not needed here and can be found in Refs.t. For the purpose of the present paper it is sufficient to know that spinors $\Phi_{j}$ can be subject to the normalization condition

$$
\int_{0}^{\pi} \int_{0}^{2 \pi}\left(\left|\Phi_{j 1}\right|^{2}+\left|\Phi_{j 2}\right|^{2}\right) \sin \vartheta d \vartheta d \varphi=1,
$$

i. e., they form an orthonormal basis in $L_{2}^{2}\left(\mathbb{S}^{2}\right)$. Further we restrict ourselves to the case $j=1$ because for $j=2,3$ the considerations are the same.

Let us employ the ansatz $F_{11}=\sqrt{\mu_{0}-\left(\omega_{1}-g A_{1}\right)} r^{\alpha_{1}} e^{-\beta_{1} r}\left[f_{11}\left(r_{1}\right)+f_{12}\left(r_{1}\right)\right]$, $F_{12}=i \sqrt{\mu_{0}+\left(\omega_{1}-g A_{1}\right)} r^{\alpha_{1}} e^{-\beta_{1} r}\left[f_{11}\left(r_{1}\right)-f_{12}\left(r_{1}\right)\right]$ with $\alpha_{1}=\sqrt{\lambda_{1}^{2}-g^{2} a_{1}^{2}}, \beta_{1}=$ $\sqrt{\mu_{0}^{2}-\left(\omega_{1}-g A_{1}\right)^{2}}, r_{1}=2 \beta_{1} r$. Then, inserting the ansatz into (25), adding and subtracting equations give rise to

$$
\begin{gathered}
\beta_{1} r_{1} f_{11}^{\prime}+Y_{1} f_{11}+Z_{1} f_{12}=0, \\
\beta_{1} r_{1} f_{12}^{\prime}-\beta_{1} r_{1} f_{12}+Y_{2} f_{12}+Z_{2} f_{11}=0,
\end{gathered}
$$

where prime signifies the differentiation with respect to $r_{1}, Y_{1,2}=\alpha_{1} \beta_{1} \mp g a_{1}\left(\omega_{1}-\right.$ $\left.g A_{1}\right), Z_{1,2}=\lambda_{1} \beta_{1} \pm g a_{1} \mu_{0}$. From (28) one yields the second order equations in $r_{1}$

$$
\begin{gathered}
r_{1} f_{11}^{\prime \prime}+\left(1+2 \alpha_{1}-r_{1}\right) f_{11}^{\prime}-\frac{Y_{1}}{\beta_{1}} f_{11}=0, \\
r_{1} f_{12}^{\prime \prime}+\left(1+2 \alpha_{1}-r_{1}\right) f_{12}^{\prime}-\left(1+\frac{Y_{1}}{\beta_{1}}\right) f_{12}=0,
\end{gathered}
$$

that are the Kummer equations (see, e. g. Ref.8) and their only solution finite at 0 and at infinity not strongly increasing is the Laguerre polynomial $L_{n_{r}^{(1)}}^{\rho}\left(r_{1}\right)$ (that always may be normalized to be equal to 1 at $r_{1}=0$ ) with $\rho=2 \alpha_{1}$ and $-n_{r}^{(1)}=Y_{1} / \beta_{1}=0,-1,-2, \ldots$ If putting

$$
f_{11}=C_{11} L_{n_{r}^{(1)}}^{\rho}\left(r_{1}\right), f_{12}=C_{12} L_{n_{r}^{(1)}-1}^{\rho}\left(r_{1}\right)
$$

then at $r_{1}=0$ from Eqs. (28) it follows $C_{11} Y_{1}+C_{12} Z_{1}=0, C_{11} Z_{2}+C_{12} Y_{2}=0$ and since $Y_{1} Y_{2}-Z_{1} Z_{2}=0$ we obtain $C_{12}=-C_{11} \frac{Y_{1}}{Z_{1}}=-C_{11} \frac{Z_{2}}{Y_{2}}$. For to describe relativistic bound states we should require $\psi \in L_{2}^{12}\left(\mathbb{R}^{3}\right)$ at any $t \in \mathbb{R}$ and one can accept the normalization condition for $F_{j 1}, F_{j 2}$ in the form

$$
\int_{0}^{\infty}\left(\left|F_{j 1}\right|^{2}+\left|F_{j 2}\right|^{2}\right) d r=\frac{1}{3}
$$

with taking into account the condition (27) so that $C_{11}$ can be determined from the relation (30). The condition $Y_{1} / \beta_{1}=-n_{r}^{(1)}=0$ signifies $Y_{1}=0$ that entails 
$Z_{1} Z_{2}=0$. Then at $\lambda_{1}>0, Z_{1}=2 g a_{1} \mu_{0}>0$ (we consider $a_{1}>0$ ), $Z_{2}=0$, $C_{12}=0$ and $n_{r}^{(1)}=0$ is admissible, i. e., $n_{r}^{(1)}=0,1,2 \ldots$. At $\lambda_{1}<0, Y_{1}=Z_{1}=0$ but $Y_{2}=2 g a_{1}\left(\omega_{1}-g A_{1}\right) \neq 0, Z_{2}=-2 g a_{1} \mu_{0} \neq 0, C_{12} \neq 0$ and $n_{r}^{(1)}=0$ is not allowed, i. e., $n_{r}^{(1)}=1,2, \ldots$. Under the situation the equality $Y_{1} / \beta_{1}=-n_{r}^{(1)}$ gives the spectrum (provided that $\omega_{1}>g A_{1}>0$ )

$$
\omega_{1}=g A_{1}+\mu_{0}\left[1+\frac{g^{2} a_{1}^{2}}{\left(n_{r}^{(1)}+\sqrt{\lambda_{1}^{2}-g^{2} a_{1}^{2}}\right)^{2}}\right]^{-1 / 2} .
$$

Further, if considering $a_{2}>0, \omega_{3}>g A_{2}>0, \omega_{2}<0$ with $\left|\omega_{2}\right|>g\left(A_{1}+A_{2}\right)$ then acting in line analogous with the above we have

$$
\begin{gathered}
\omega_{3}=g A_{2}+\mu_{0}\left[1+\frac{g^{2} a_{2}^{2}}{\left(n_{r}^{(3)}+\sqrt{\lambda_{3}^{2}-g^{2} a_{2}^{2}}\right)^{2}}\right]^{-1 / 2}, \\
\omega_{2}=-g\left(A_{1}+A_{2}\right)-\mu_{0}\left[1+\frac{g^{2}\left(a_{1}+a_{2}\right)^{2}}{\left(n_{r}^{(2)}+\sqrt{\lambda_{2}^{2}-g^{2}\left(a_{1}+a_{2}\right)^{2}}\right)^{2}}\right]^{-1 / 2},
\end{gathered}
$$

and the spectrum of meson is obtained in the form

$$
\begin{gathered}
\frac{\varepsilon}{\mu_{0}}=\left[1+\frac{g^{2} a_{1}^{2}}{\left(n_{r}^{(1)}+\sqrt{\lambda_{1}^{2}-g^{2} a_{1}^{2}}\right)^{2}}\right]^{-1 / 2}-\left[1+\frac{g^{2}\left(a_{1}+a_{2}\right)^{2}}{\left(n_{r}^{(2)}+\sqrt{\lambda_{2}^{2}-g^{2}\left(a_{1}+a_{2}\right)^{2}}\right)^{2}}\right]^{-1 / 2} \\
+\left[1+\frac{g^{2} a_{2}^{2}}{\left(n_{r}^{(3)}+\sqrt{\lambda_{3}^{2}-g^{2} a_{2}^{2}}\right)^{2}}\right]^{-1 / 2}
\end{gathered}
$$

where the numbers $n_{r}^{(2,3)}$ are subject to the same conditions as for $n_{r}^{(1)}$.

\section{Influence of the Dirac-like monopole configurations of gluonic field}

If $K_{j} \neq 0$ then according to the results of Refs 1 we shall get the similar relations providing that $K_{j}=k_{j} / g$ with $k_{j} \in \mathbb{Z}$, i. e., $k_{j}$ are integer numbers. But now we should consider the spinor $\Phi_{j}$ of (22) to be the eigenspinor $\Phi_{j}$ of the twisted euclidean Dirac operator $\mathcal{D}_{k}$ on the unit sphere $\mathbb{S}^{2}$, respectively, with the Chern numbers $k=k_{1},-\left(k_{1}+k_{2}\right), k_{2}$ and the eigenvalues $\lambda_{j}$ should be, accordingly, replaced by $\lambda_{1}= \pm \sqrt{\left(l_{1}+1\right)^{2}-k_{1}^{2}}, l_{1} \geq\left|k_{1}\right|, \lambda_{3}= \pm \sqrt{\left(l_{3}+1\right)^{2}-k_{2}^{2}}$, $l_{3} \geq\left|k_{2}\right|, \lambda_{2}= \pm \sqrt{\left(l_{2}+1\right)^{2}-\left(k_{1}+k_{2}\right)^{2}}, l_{2} \geq\left|k_{1}+k_{2}\right|$ (for more details see Refs. f) ). Physically the corresponding configurations of gluonic field describe the Dirac-like monopole ones with magnetic charges, conformably, $P_{1}=k_{1} / g, P_{2}=-\left(k_{1}+k_{2}\right) / g$, $P_{3}=k_{2} / g$ but the total (nonabelian) magnetic charge of the given configurations remains equal to $P_{1}+P_{2}+P_{3}=0$. A number of authors (in particular, those who develop lattice approach) insist on important role of such configurations in a possible mechanism of the quark confinement (see, e. g. Refs. 6 and references therein). 


\section{Spectrum of bound states in the Coulomb-linear case}

We can get the approximate solutions of Eqs. (12)-(14) if considering $\sigma_{2} \Phi_{j}=\Phi_{j}$ for the eigenspinor $\Phi_{j}$ of (22). As follows from explicit form of those spinors found in Refs. 1 this can be fulfilled only approximately and it is clear that when doing so we make an error retaining eigenvalues $\lambda_{j}$ of the euclidean Dirac operator $\mathcal{D}_{0}$

on the unit sphere $\mathbb{S}^{2}$ instead of the eigenvalues of a less symmetric operator on $\mathbb{S}^{2}$ whose form is unknown explicitly. As will be seen below, however, this may seemingly be compensated by the choice of parameters $B_{j}$ of models so that the solutions obtained further may be considered as the almost exact ones. Let us employ the solutions of (16) and (21) for the Yang-Mills equations (7) with the source $J$ described late in Sec. 3 where at first we put $K_{j}=0$ as well. Using the given solutions and the ansatz (22) we shall, in line with the above, get, e. g. from (12), the system (23) which (having accepted $\sigma_{2} \Phi_{j} \approx \Phi_{j}$ ) yields

$$
\begin{gathered}
{\left[\partial_{r}+\frac{\lambda_{1}}{r}-g\left(b_{1}+\frac{B_{1}}{r}\right)\right] F_{11}=i\left(\mu_{0}-c_{1}\right) F_{12},} \\
{\left[\partial_{r}-\frac{\lambda_{1}}{r}+g\left(b_{1}+\frac{B_{1}}{r}\right)\right] F_{12}=-i\left(\mu_{0}+c_{1}\right) F_{11} .}
\end{gathered}
$$

Now we employ the ansatz $F_{11}=A r^{\alpha_{1}} e^{-\beta_{1} r}\left[f_{11}\left(r_{1}\right)+f_{12}\left(r_{1}\right)\right], F_{12}=i B r^{\alpha_{1}} e^{-\beta_{1} r}\left[f_{11}\left(r_{1}\right)-\right.$ $\left.f_{12}\left(r_{1}\right)\right]$ with $\alpha_{1}=\sqrt{\left(\lambda_{1}-g B_{1}\right)^{2}-g^{2} a_{1}^{2}}, \beta_{1}=\sqrt{\mu_{0}^{2}-\left(\omega_{1}-g A_{1}\right)^{2}+g^{2} b_{1}^{2}}, A=$ $g b_{1}+\beta_{1}, B=\mu_{0}+\omega_{1}-g A_{1}, r_{1}=2 \beta_{1} r$. After this, inserting the ansatz into (34), adding and subtracting equations entail

$$
\begin{gathered}
r_{1} A B f_{11}^{\prime}+Y_{1} f_{11}+Z_{1} f_{12}=0 \\
r_{1} A B f_{12}^{\prime}-r_{1} A B f_{12}+Y_{2} f_{12}+\left(Z_{2}-\frac{g b_{1}}{\beta_{1}} A B r_{1}\right) f_{11}=0,
\end{gathered}
$$

where prime signifies the differentiation with respect to $r_{1}, Y_{1,2}=\left[\alpha_{1} \beta_{1} \mp g a_{1}\left(\omega_{1}-\right.\right.$ $\left.\left.\left.g A_{1}\right)+g \alpha_{1} b_{1}\right] B \pm g^{2} a_{1} b_{1} A, Z_{1,2}=\left[\left(\lambda_{1}-g B_{1}\right) A \pm g a_{1} \mu_{0}\right)\right] B \pm g^{2} a_{1} b_{1} A$ and $Y_{1} Y_{2}-$ $Z_{1} Z_{2}=0$.

From $\left(35^{\prime}-35^{\prime \prime}\right)$ one yields the second order equations in $r_{1}$

$$
\begin{gathered}
r_{1} f_{11}^{\prime \prime}+\left(1+2 \alpha_{1}-r_{1}\right) f_{11}^{\prime}+n_{r}^{(1)} f_{11}=0 \\
r_{1} f_{12}^{\prime \prime}+\left(\frac{a}{a-b r_{1}}+2 \alpha_{1}-r_{1}\right) f_{12}^{\prime}+n_{r}^{(1)}\left(\frac{a \kappa}{a-b r_{1}}+1\right) f_{12}=0
\end{gathered}
$$

with $a=Z_{2}, b=g b_{1} A B / \beta_{1}, \kappa=A B / Y_{1}$ and

$$
n_{r}^{(1)}=\frac{g b_{1} Z_{1}-\beta_{1} Y_{1}}{\beta_{1} A B} .
$$

It is clear that according to (36) we should choose $f_{11} \sim L_{n_{r}^{(1)}}^{\rho}\left(r_{1}\right)$ with the Laguerre polynomial $L_{n_{r}^{(1)}}^{\rho}\left(r_{1}\right)\left(\rho=2 \alpha_{1}\right)$ if $n_{r}^{(1)}=0,1,2 \ldots$ 
A little bit below we shall show that one can choose $f_{12}\left(r_{1}\right) \sim P_{n_{r}^{(1)}}^{\rho}\left(r_{1}\right)$ with some polynomial $P_{n_{r}^{(1)}}^{\rho}\left(r_{1}\right)$, consequently, we shall gain that $\psi_{1} \in L_{2}^{4}\left(\mathbb{R}^{3}\right)$ at any $t \in \mathbb{R}$ and, as a result, we obtain from (38) that $\omega_{1}$ should be determined from the equation

$$
\begin{gathered}
{\left[g^{2} a_{1}^{2}+\left(n_{r}^{(1)}+\alpha_{1}\right)^{2}\right]\left(\omega_{1}-g A_{1}\right)^{2}+2\left(\lambda_{1}-g B_{1}\right) g^{2} a_{1} b_{1}\left(\omega_{1}-g A_{1}\right)+} \\
{\left[\left(\lambda_{1}-g B_{1}\right)^{2}-\left(n_{r}^{(1)}+\alpha_{1}\right)^{2}\right] g^{2} b_{1}^{2}-\mu_{0}^{2}\left(n_{r}^{(1)}+\alpha_{1}\right)^{2}=0}
\end{gathered}
$$

that yields

$$
\omega_{1}=g A_{1}-\frac{\left(\lambda_{1}-g B_{1}\right) g^{2} a_{1} b_{1}-\sqrt{X_{1}}}{g^{2} a_{1}^{2}+\left(n_{r}^{(1)}+\alpha_{1}\right)^{2}}
$$

with $X_{1}=\left(\lambda_{1}-g B_{1}\right)^{2} g^{4} a_{1}^{2} b_{1}^{2}-\left[g^{2} a_{1}^{2}+\left(n_{r}^{(1)}+\alpha_{1}\right)^{2}\right]\left\{\left[\left(\lambda_{1}-g B_{1}\right)^{2}-\left(n_{r}^{(1)}+\alpha_{1}\right)^{2}\right] g^{2} b_{1}^{2}-\right.$ $\left.\mu_{0}^{2}\left(n_{r}^{(1)}+\alpha_{1}\right)^{2}\right\}$ and the sign of $\sqrt{X_{1}}$ is chosen from the fact that the expression (40) should pass on to (31) at $b_{1}=B_{1}=0$. The similar relations will also hold true for $\omega_{2,3}$ with replacing $a_{1}, A_{1}, b_{1}, B_{1} \rightarrow a_{2}, A_{2}, b_{2}, B_{2}$ respectively for $\omega_{3}$ and $a_{1}, A_{1}, b_{1}, B_{1} \rightarrow-\left(a_{1}+a_{2}\right),-\left(A_{1}+A_{2}\right),-\left(b_{1}+b_{2}\right),-\left(B_{1}+B_{2}\right)$ respectively for $\omega_{2}$ and the spectrum of meson will be

$$
\begin{gathered}
\varepsilon=-\frac{\left(\lambda_{1}-g B_{1}\right) g^{2} a_{1} b_{1}-\sqrt{X_{1}}}{g^{2} a_{1}^{2}+\left(n_{r}^{(1)}+\alpha_{1}\right)^{2}} \\
-\frac{\left[\lambda_{2}+g\left(B_{1}+B_{2}\right)\right] g^{2}\left(a_{1}+a_{2}\right)\left(b_{1}+b_{2}\right)+\sqrt{X_{3}}}{g^{2}\left(a_{1}+a_{2}\right)^{2}+\left(n_{r}^{(2)}+\alpha_{2}\right)^{2}}-\frac{\left(\lambda_{3}-g B_{2}\right) g^{2} a_{2} b_{2}-\sqrt{X_{2}}}{g^{2} a_{2}^{2}+\left(n_{r}^{(3)}+\alpha_{3}\right)^{2}},
\end{gathered}
$$

where $\alpha_{2,3}$ and $X_{2,3}$ are obtained from $\alpha_{1}, X_{1}$ by the same above replacements while the signs of $\sqrt{X_{j}}$ are chosen to correspond to the expression (33) in the Coulomblike case $b_{j}=B_{j}=0$. We can see that all dependence of $\lambda_{j}$ is expressed through the combinations $\lambda_{j} \pm g B_{j}$ and the approximation error mentioned early in this section may probably be compensated by choosing $B_{j}$ in a proper way.

Now let us describe the above polynomial $P_{n_{r}^{(1)}}^{\rho}\left(r_{1}\right)$ which should be the solution of the equation (37). Instead of solving (37), however, we use the fact that the sought polynomial should also obey the first order linear equation $\left(35^{\prime \prime}\right)$ with $f_{11}\left(r_{1}\right)=$ $L_{n_{r}^{(1)}}^{\rho}\left(r_{1}\right)$ which allows us to write down $P_{n_{r}^{(1)}}^{\rho}\left(r_{1}\right)$ in the form (putting $n_{r}^{(1)} \equiv n$, $r_{1} \equiv x$ for brevity)

$$
P_{n}^{\rho}(x)=x^{-\alpha} e^{x} \int_{0}^{x} t^{\alpha}\left(\frac{g b_{1}}{\beta_{1}}-\frac{Z_{2}}{A B t}\right) e^{-t} L_{n}^{\rho}(t) d t
$$

with $\alpha=2 \alpha_{1}+n-g b_{1} Z_{1} /\left(\beta_{1} A B\right)$. Further we can employ the relation 10

$$
\int_{0}^{x} t^{\alpha-1} e^{-t} L_{n}^{\rho}(t) d t=\frac{x^{\alpha}(1+\rho)_{n}}{n ! \alpha}{ }_{2} F_{2}(\alpha, 1+\rho+n ; \alpha+1,1+\rho ;-x), \operatorname{Re} \alpha, x>0
$$

with the generalized hypergeometric function ${ }_{2} F_{2}$ of order $(2,2)$ while the Pohgammer symbol $(a)_{k}=a(a+1) \ldots(a+k-1)=\Gamma(a+k) / \Gamma(a)$ with the Euler gamma 
function $\Gamma(z)$, the relation 11

${ }_{2} F_{2}(\alpha, 1+\rho+n ; \alpha+1,1+\rho ;-x)=\sum_{k=0}^{n} \frac{(-1)^{k} x^{k}}{(1+\rho)_{k}} C_{n}^{k} \frac{(\alpha)_{k}}{(\alpha+1)_{k}} \Phi(\alpha+k, \alpha+k+1 ;-x)$

with the Kummer function $\Phi \equiv{ }_{1} F_{1}$ and the relation $8(z, z+1 ;-x)=z x^{-z} \gamma(z, x)$ with the incomplete gamma function $\gamma(z, x)$ to come to the expression

$P_{n}^{\rho}(x)=\frac{(1+\rho)_{n}}{n !} x^{-\alpha} e^{x}\left\{\sum_{k=0}^{n} \frac{(-1)^{k}}{(1+\rho)_{k}} C_{n}^{k}\left[\frac{g b_{1}}{\beta_{1}} \gamma(\alpha+k+1, x)-\frac{Z_{2}}{A B} \gamma(\alpha+k, x)\right]\right\}$.

Now, using the functional equation $\gamma(z+1)=z \gamma(z)-x^{z} e^{-x}$, it is not complicated to see that $\gamma(\alpha+k+1, x)=(\alpha+k) \gamma(\alpha+k)-x^{\alpha+k} e^{-x}, \gamma(\alpha+k, x)=(\alpha)_{k} \gamma(\alpha, x)-$ $\left[\sum_{i=1}^{k}(\alpha+i)_{k-i} x^{i-1}\right] x^{\alpha} e^{-x}$ and then one can show with the help of (38) and of equality $Y_{1} Y_{2}-Z_{1} Z_{2}=0$ that

$$
\frac{(1+\rho)_{n}}{n !} \sum_{k=0}^{n} \frac{(-1)^{k}}{(1+\rho)_{k}} C_{n}^{k}\left[\frac{g b_{1}}{\beta_{1}}(\alpha+k)-\frac{Z_{2}}{A B}\right](\alpha)_{k}=0 .
$$

At last, employing the relation 12

$$
\frac{(1+\rho)_{n}}{n !} \sum_{k=0}^{n} \frac{(-1)^{k} x^{k}}{(1+\rho)_{k}} C_{n}^{k}=L_{n}^{\rho}(x),
$$

we come to the final form of the sought polynomial

$$
\begin{gathered}
P_{0}^{\rho}(x)=-\frac{g b_{1}}{\beta_{1}} L_{0}^{\rho}(x)=-\frac{g b_{1}}{\beta_{1}} \\
P_{n}^{\rho}(x)=-\frac{(1+\rho)_{n}}{n !} \sum_{k=1}^{n} \frac{(-1)^{k}}{(1+\rho)_{k}} C_{n}^{k}\left[\frac{g b_{1}}{\beta_{1}}(\alpha+k)-\frac{Z_{2}}{A B}\right]\left[\sum_{i=1}^{k}(\alpha+i)_{k-i} x^{i-1}\right] \\
-\frac{g b_{1}}{\beta_{1}} L_{n}^{\rho}(x), n=1,2,3 \ldots
\end{gathered}
$$

Accordingly, we can now put $f_{11}=C_{11} L_{n}^{\rho}(x), f_{12}=C_{12} P_{n}^{\rho}(x)$ and from the system $\left(35^{\prime}-35^{\prime \prime}\right)$ at $r_{1} \equiv x=0$ it follows the relation between the constants $C_{11}, C_{12}$

$$
C_{11}=-\frac{Z_{1}}{Y_{1}} \frac{P_{n}^{\rho}(0)}{L_{n}^{\rho}(0)} C_{12}=-\frac{Y_{2}}{Z_{2}} \frac{P_{n}^{\rho}(0)}{L_{n}^{\rho}(0)} C_{12} .
$$

For completeness let us adduce the normalized radial part of $\psi_{1}$-component for the meson wave function of ground state (where $n=0$ and then $C_{11}=C_{12}=C$ )

$$
\begin{gathered}
F_{11}=C r^{\alpha_{1}} e^{-\beta_{1} r}\left(1-\frac{g b_{1}}{\beta_{1}}\right)\left(g b_{1}+\beta_{1}\right), \\
F_{12}=i C r^{\alpha_{1}} e^{-\beta_{1} r}\left(1+\frac{g b_{1}}{\beta_{1}}\right)\left(\mu_{0}+\omega_{1}-g A_{1}\right),
\end{gathered}
$$


where $C$ is determined from the relation (30) with the help of formula 12 $\int_{0}^{\infty} x^{\alpha-1} e^{-p x} d x=$ $\Gamma(\alpha) p^{-\alpha}, \operatorname{Re} \alpha, p>0$, which entails

$C^{2}\left[\left(1-\frac{g b_{1}}{\beta_{1}}\right)^{2}\left(g b_{1}+\beta_{1}\right)^{2}+\left(1+\frac{g b_{1}}{\beta_{1}}\right)^{2}\left(\mu_{0}+\omega_{1}-g A_{1}\right)^{2}\right] \Gamma\left(2 \alpha_{1}+1\right)\left(2 \beta_{1}\right)^{-\left(2 \alpha_{1}+1\right)}=\frac{1}{3}$.

It is evident that the similar expressions will hold true for the corresponding radial parts of $\psi_{2,3}$-components with taking into account the replacements described above.

Finally, it should be noted that the influence of the Dirac-like monopole configurations for gluonic field (when $K_{j} \neq 0$ ) can be treated by the same manner as in Sec. 5 if taking $\sigma_{2} \Phi_{j} \approx \Phi_{j}$ for the eigenspinor $\Phi_{j}$ of the twisted euclidean Dirac operator $\mathcal{D}_{k}$ on the unit sphere $\mathbb{S}^{2}$ with the conforming Chern numbers $k=k_{1},-\left(k_{1}+k_{2}\right), k_{2}$.

\section{Concluding remarks}

So we have seen that the black hole physics methods and results may really help to build relativistic models of mesons that are parametrized by constants $\mu_{0}, g, a_{j}, A_{j}, b_{j}, B_{j}, K_{j}$ which should evidently be determined by comparing to experimental data. Even in the case of pure Coulomb interaction these models give essentially different spectrum of bound states in comparison with the naive picture of threefold positronium-like spectrum accepted, e. g., in modern quarkonium spectroscopy. It seems to us that the exact equations derived in the paper may serve as a basis to construct miscellaneous approximate approaches in the spirit of that of Sec. 6. We hope to continue further study of the questions raised here.

\section{Acknowledgements}

The work was supported in part by the Russian Foundation for Basic Research (grants Nos. 98-02-18380-a and 01-02-17157) and by GRACENAS (grant No. 6-181997).

\section{References}

1. T. Feldmann, Int. J. Mod. Phys. A15, 159 (2000).

2. J. Carbonell, B. Desplanques, V. A. Karmanov, J.-F. Mathiot, Phys. Rep. 300, 215 (1998);

S. Brodsky, H-C. Pauli, S. Pinsky, Phys. Rep. 301, 299 (1998).

3. Yu. P. Goncharov, Nucl. Phys. B460, 167 (1996); Int. J. Mod. Phys. A12, 3347 (1997); Pis'ma v ZhETF 67, 1021 (1998); Mod. Phys. Lett. A13, 1495 (1998).

4. Yu. P. Goncharov, Pis'ma v ZhETF 69, 619 (1999); Phys. Lett. B458, 29 (1999).

5. Géométrie Riemannian en Dimension 4. Seminaire Arthur Besse (Cedic/Fernand Nathan, 1981).

6. H. B. Lawson, Jr. and M.-L. Michelsohn, Spin Geometry (Princeton U. P., 1989).

7. A. L. Besse, Einstein Manifolds (Springer-Verlag, 1987);

M. M. Postnikov, Riemannian Geometry (Factorial, 1998). 
8. Handbook of Mathematical Functions. Eds. M. Abramowitz and I. A. Stegun (National Bureau of Standards, 1964).

9. H. Reinhardt, Nucl. Phys. Proc. Suppl. 74, 364 (1999);

A. Di Giacomo, Prog. Theor. Phys. Suppl. 131, 161 (1998); Nucl. Phys. A661, 13 (1999);

M. Fukushima, H. Suganuma and H. Toki, Phys. Rev. D60, 094504 (1999).

10. A. P. Prudnikov, Yu. A. Brychkov and O. I. Marichev, Integrals and Series. Special Functions (Nauka, 1983).

11. A. P. Prudnikov, Yu. A. Brychkov and O. I. Marichev, Integrals and Series. Additional Chapters (Nauka, 1986).

12. A. P. Prudnikov, Yu. A. Brychkov and O. I. Marichev, Integrals and Series. Elementary Functions (Nauka, 1981).

13. V. V. Dvoeglazov, R. N. Faustov and Yu. N. Tukhtyaev, Fiz. Elem. Chast. i Atom. Yad. 25, 144 (1994). 